\section{Observational studies and the chicken and egg issue in stroke}

\author{
Tara Purvis and Dominique A. Cadilhac
}

In a News \& Views article (Are care and outcomes better for participants of stroke trials? Nat. Rev. Neurol. 12, 498-499; 2016) ${ }^{1}$, Mary Joan Macleod and Carl E. Counsell commented on our recent publication ${ }^{2}$, in which patients with stroke who participated in research were found to receive better care and experience fewer in-hospital deaths than patients not participating in research. We thank Dr Macleod and Dr Counsell for their interest in our article, and for their comments, which highlighted the limitations of observational studies for exploring this topic. Overall, the important message was that research participants appear to have a greater likelihood of receiving care in stroke units. Therefore, are the observed benefits attributable to participation in research, or to the fact that being on a stroke unit - which in itself is known to improve outcomes - increases the opportunity to take part in research studies?

We agree that it was not possible for us to adjust for all potential confounders, as is often the case in nonrandomized studies. In particular, we were unable to adjust for the fact that patients who participate in research are often a prognostically more favourable group, as poor health and existing comorbidities generally preclude patients from participating in research with long-term follow-up.
The likelihood of residual confounding was clearly stated in our discussion. Although the odds ratio for in-hospital death was large following adjustment for the available confounders, the imprecision of this result was apparent from the wide confidence intervals reported in our paper. This level of uncertainty guided our conservative conclusion that those who participate in research "potentially have improved in-hospital survival."

Macleod and Counsell highlighted the fact that some trials can increase the risk of harm, and noted two randomized controlled trials (RCTs) that were halted early due to this risk ${ }^{1}$. We agree that this situation can arise, and that adverse events need to be closely monitored. However, our definition of research participation was not restricted to RCTs. As explained, the type of study to which the patient consented was unknown. Therefore, it is possible that some patients in our study were participating in observational studies or translational research, where research participation might have little or no bearing on the risk of an adverse outcome. Furthermore, early termination of RCTs is not always due to adverse outcomes. A recent endovascular clot retrieval trial was ended by the Data and Safety Monitoring Committee owing to the loss of equipoise ${ }^{3}$ in light of significant reductions in disability from similar trials that had finished. In addition, the limited number of patients with acute stroke enrolled in research (5\%) that we reported on may be an artefact of the limited number and type of hospitals that offer research (30\% in 2011, $41 \%$ in 2013). Therefore, further to improving participation rates of patients in research, there is also scope for more Australian hospitals to be involved in research.

It was evident that patients who participated in research were more likely to be treated in a stroke unit, which will have contributed to an increased likelihood of receiving a range of different evidence-based (process-of-care) interventions ${ }^{4,5}$. In response to the commentary by Macleod and Counsell, we performed an additional sensitivity analysis, using multi-level random effects logistic regression, to adjust for the effects of stroke unit care and patient clustering at the hospital level on the adherence to processes of care. Following this level of adjustment, greater access to allied health and other acute treatments such as treatment with thrombolysis were still more likely to be provided to research participants than to nonparticipants in our cohort (TABLE 1). Access to stroke unit care in Australia remains suboptimal, with only $67 \%$ of patients treated in a stroke unit according to the 2015 National Audit ${ }^{6}$. It was not evident whether being treated in a stroke unit increased the likelihood of being considered for research or, conversely, whether patients recruited to a research study were more likely to get access to stroke units. Regardless of the reason, the greater likelihood of stroke unit care among research participants should be considered

Table 1 | Comparison of univariate and multilevel regression analyses

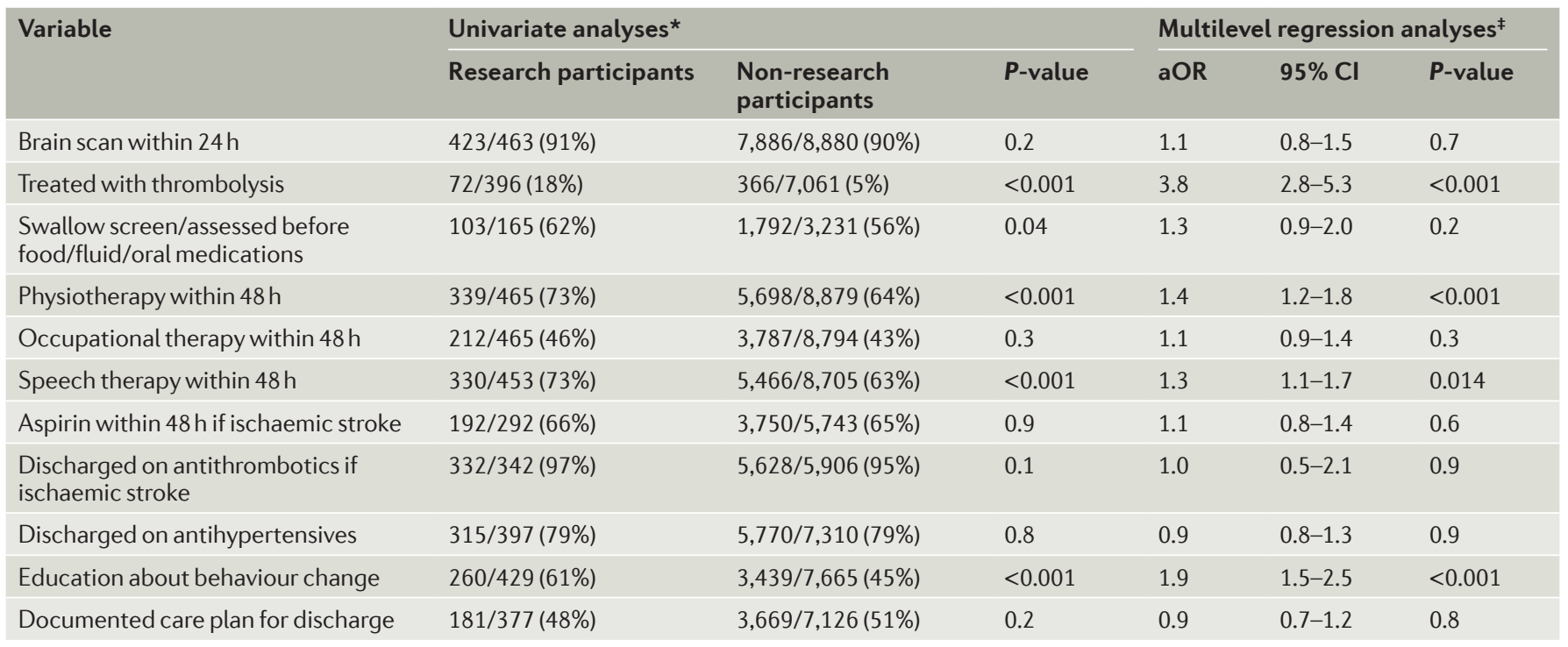

*As reported in Purvis et al. ${ }^{2} .{ }^{\ddagger}$ Outcome in this model was individual process of care; data were adjusted for receiving care in a stroke unit. aOR, adjusted odds ratio. 
clinically meaningful, as stroke units are the most universally applicable intervention known to improve outcome after stroke.

Importantly, we agree with the comment from Macleod and Counsell that it cannot automatically be assumed that taking part in clinical trials benefits all patients, and that patients should not be told that doing so will improve their outcomes. The importance of participants being well informed about potential adverse effects, particularly in the case of novel interventions, cannot be overstated. However, our results, which were based on a national sample, including both organizational and clinical data, do provide support for the notion that those patients who participate in research, irrespective of whether they are randomly assigned to control groups or involved in observational studies, are likely to receive increased access to recommended care such as stroke units, which can provide close monitoring and access to a range of health-care professionals for rehabilitation. As stated, we also agree that a need exists for further welldesigned research in this area using larger samples of patients with stroke.
Tara Purvis and Dominique A. Cadilhac are at the Translational Public Health and Evaluation Division,

Stroke and Ageing Research, School of Clinical Sciences at Monash Health, Monash University, Level 1/43-51 Kanooka Grove, Clayton, Victoria 3168, Australia.

Correspondence to T.P. tara.purvis@monash.edu

1. Macleod, M. J. \& Counsell, C. E. Stroke: are care and outcomes better for participants of stroke trials? Nat. Rev. Neurol. 12, 498-499 (2016).

2. Purvis, T., Hill, K., Kilkenny, M., Andrew, N. \& Cadilhac, D. Improved in-hospital outcomes and care for patients in stroke research: an observational study. Neurology 87, 206-213 (2016).

3. Jovin, T. G. et al. Thrombectomy within 8 hours after symptom onset in ischemic stroke. N. Engl. J. Med. 372, 2296-2306 (2015).

4. Cadilhac, D. A., Pearce, D. C., Levi, C. R. \& Donnan, G. A. Improvements in the quality of care and health outcomes with new stroke care units following implementation of a clinician-led, health-system redesign programme in New South Wales, Australia. Qual. Saf. Health Care 17, 329-333 (2008).

5. Stroke Unit Trialists' Collaboration. Organised inpatient (stroke unit) care for stroke. Cochrane Database Syst. Rev. 9, CD000197 (2013).

6. National Stroke Foundation. National Stroke Audit Acute Services Report 2015. StrokeFoundation.au https://informme.org.au/-/media/ A3039E82D9FF4BC7803B5C67B42DBBB0. ashx?la =en (2015)

Competing interests statement

The authors declare no competing interests. 